\title{
Design of a tomographic hard X-ray spectrometer for suprathermal electron studies with ECRH
}

\author{
S. Gnesin and S. Coda \\ Ecole Polytechnique Fédérale de Lausanne (EPFL), Centre de Recherches en Physique des Plasmas, \\ Association Euratom-Confédération Suisse, CH-1015 Lausanne, Switzerland.
}

\begin{abstract}
Electron cyclotron resonance heating (ECRH) and current drive (ECCD), disruptive instability events and sawtooth activity have been demonstrated to produce suprathermal electrons in fusion devices. The importance of these phenomena for fusion reactors renders suprathermal electron generation and dynamics a key topic in the physics of burning plasmas. Here some significant results from the TCV tokamak are briefly reviewed and a preliminary design of a novel tomographic hard X-ray spectrometer proposed for TCV is discussed. The design is aided by simulations of tomographic reconstruction.
\end{abstract}

Keywords: Hard -X-rays, suprathermal electrons, tomography, spectrometer, ECRH, ECCD. PACS: $52.70 . \mathrm{La}, 52.50 . \mathrm{Sw}, 52.55 \mathrm{Wq}$

\section{Introduction}

The physics of electron cyclotron current drive (ECCD) ${ }^{1}$ is intimately tied with the dynamics of suprathermal electrons, which remains only partly understood in presentday magnetic-confinement devices. An increased understanding of the suprathermal electron dynamics can have direct implications for the first experimental fusion reactor currently under construction, ITER. Indeed, ECCD is planned to be used as a tool in ITER for MHD stability control'; additionally, sawtooth activity and disruptive instabilities, which are also accompanied by substantial suprathermal electron generation, are expected to occur in the ITER baseline regime. The development of increasingly advanced fast-electron diagnostics is therefore important in view of the next generation of burning-plasma devices.

\section{Brief Review of the TCV Results}

The TCV (Tokamak à Configuration Variable) plasma is routinely heated by means of ECRH; moreover ECCD is extensively used ${ }^{3}$ to tailor the current profile for applications ranging from fully non-inductive operation to high-performance scenarios with electron internal transport barriers. With ECCD a suprathermal electron population is generated in an energy range from a few tens to a few hundred $\mathrm{keV}$, resulting in hard X-ray (HXR) bremsstrahlung emission. Studies have been conducted on TCV with a single, multichordal spectroscopic HXR camera (on loan from Tore Supra intermittently between 1999 and 2005) ${ }^{4}$ and with a high-field-side electron cyclotron emission (ECE) radiometer. These studies have led in particular to the recognition of the role of the spatial transport of suprathermal electrons, at high power densities, in regulating the magnitude and spatial distribution of the driven current ${ }^{3}$. The temporal dynamics of the suprathermal population has been investigated in detail with non-perturbative pulsed ECCD experiments combined with coherent averaging of the ECE signals ${ }^{6}$. More recent measurements of oblique ECE have provided evidence

CP988, Buming Plasma Diagnostics, edited by F. P. Orsitto, G. Gorini, E. Sindoni, and M. Tardochi (C) 2008 American Institute of Physics 978-0-7354-0507-3/08/ $\$ 23.00$ 
of distribution function asymmetries with $\mathrm{ECCD}^{7}$. Additionally, the absorption of third-harmonic ECRH on TCV has been shown to be significantly enhanced by the presence of a fast-electron tail ${ }^{8}$. Evidence of the generation and subsequent rapid cross-field transport of suprathermal electrons during sawtooth crashes has also emerged from ECE measurements ${ }^{9}$.

\section{Proposed tomographic spectroscopic system for TCV}

Experimental evidence as well as theoretical predictions indicate that poloidal asymmetries exist in the HXR emissivity. A natural next step is therefore the development of tomographic HXR systems to investigate the full 2D emissivity distribution in the poloidal plane. For these reasons we have carried out a preliminary design of a new tomographic set of two to nine spectroscopic poloidal HXR cameras for the TCV geometry, with broad coverage of the poloidal cross section and very high spatial and temporal resolution. The proposed system is based on a multi-camera set of fan views which have been conceived to provide high flexibility and compactness, as required by the constraints of the TCV vessel environment. We envision using CdTe semiconductor diodes, which do not require bulky cooling or magnetic-shielding equipment and retain acceptable energy resolution (typically $5-10 \mathrm{keV})^{4,10}$. The HXR radiation is channeled to the detectors by a novel tungsten collimator configuration, adapted from the Soller collimator concept ${ }^{11}$, and compatible with an extremely compact camera design. In this configuration the collimator plates are arranged in a fan and each plate defines the aperture and provides the necessary shielding against oblique illumination (see Fig. 1). The design of the collimator aperture can be adjusted for each individual detector in order to determine the étendue and the attendant chord spread in the plasma region. The spatial sampling properties of the system are determined by the typical chord separation in the plasma region, $\sim 2 \mathrm{~cm}(8 \%$ of the minor radius) in our design.
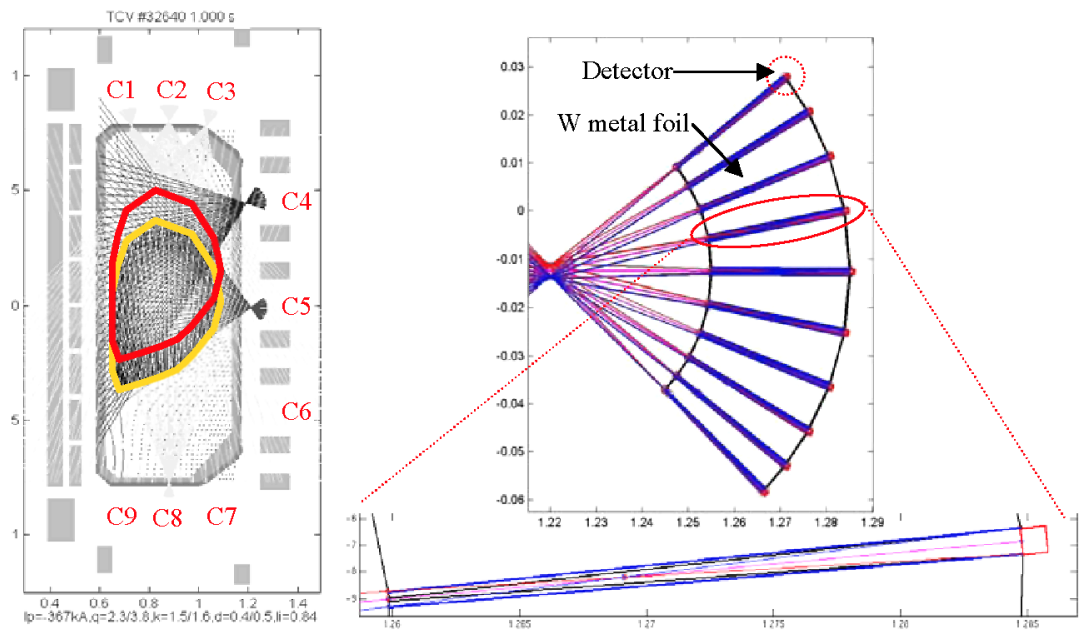

FIGURE 1. Chord fan coverage for the hard X-ray camera system in TCV (left); detail of the collimator system in camera 5 (right). Possible cameras are numbered from 1 to 9 , corresponding to the nine ports seen in the figure, in clockwise order from the top left. 
The detectors can be placed immediately behind the Soller plates, enabling them to be housed directly within the port opening. The extreme compactness and flexibility of this design should make it readily adaptable to other fusion devices.

\section{Tomographic simulations and validation}

The number and distribution of the cameras is determined by a compromise between the quality of tomographic reconstruction and cost, within the constraints set by the TCV port geometry. In order to base the choice on a quantitative foundation, we have performed simulations to compare imposed ("phantom") HXR emissivity patterns with the tomographic inversions resulting from the calculated detector signal distributions in different configurations. Different emission patterns have been used in the analysis: poloidally-symmetric emissivity profiles reconstructed from past, nontomographic HXR measurements during ECRH in TCV, as well as periodic poloidal distributions with a poloidal wave number $m$, to simulate increasingly complex poloidal asymmetries. The tomographic reconstruction algorithm performs a line integration of the simulated photon emissivity $g(R, z)$ along the i-detector's line of sight $\left(\mathrm{L}_{i}\right)$ in order to obtain the so called chord brightness $f_{i}=\int_{L i} g(R, z) d l$. This information is thus fed into the tomographic algorithm that generates the reconstructed plasma emissivity on a pixel grid using the minimum Fisher information method in order to condition the solution $^{12}$. The simulations show the expected (see Fig. 2) increase in the quality of the tomographic reconstruction when cameras viewing the plasma from maximally separated directions are added. As a general rule, $\mathrm{m}+\mathbf{l}$ independent views are required to recover an emissivity pattern with wave number $\mathrm{m}$. This is confirmed by Fig. 2 for the cases $m=0,1$, and 2 . The algorithm used in this study enables one to recover the main features (peak positions and relative intensity distribution) of the plasma emissivity; but the robustness in reconstructing emissive patterns with high spatial gradients must still be improved. Further work will be devoted to the upgrade and comparison of the present algorithm with alternative ones (maximum entropy and linear regularization). A final quantitative assessment of optimal camera orientation and distribution is expected to come in the near future.

\section{Acknowledgment}

This work was supported in part by the Swiss National Science Foundation.

\section{References}

1. N.J. Fisch, Rev. Mod. Phys. 59, 175 (1987).

2. R.J. La Haye et al, Nucl. Fusion 46, 451 (2006).

3. S. Coda et al, Nucl. Fusion. 43, 1361 (2003).

4. Y. Peysson, S. Coda, F. Imbeaux, Nucl. Inst. Meth. A 458, 269 (2001).

5. P. Blanchard et al, Plasma Phys. Control. Fusion 44, 2231 (2002).

6. S. Coda et al, Plasma Phys. Control. Fusion 48, B359 (2006).

7. T.P. Goodman et al, Proc. 34th EPS Conf. on Control. Fusion and Plasma Phys., Warsaw, Poland, 2007 (P2.147).

8. S. Alberti et al, Nucl. Fusion 42, 42 (2002).

9. I. Klimanov et al, Plasma Phys. Control. Fusion 49, Ll (2007).

10.T. Takahashi and S. Watanabe, IEEE Trans. Nucl. Sci. 48, 950 (2001).

11.W. Soller, Phys. Rev. 24, 158 (1924). 
12. M. Anton et al, Plasma Phys. Control. Fusion 38, 1849 (1996).
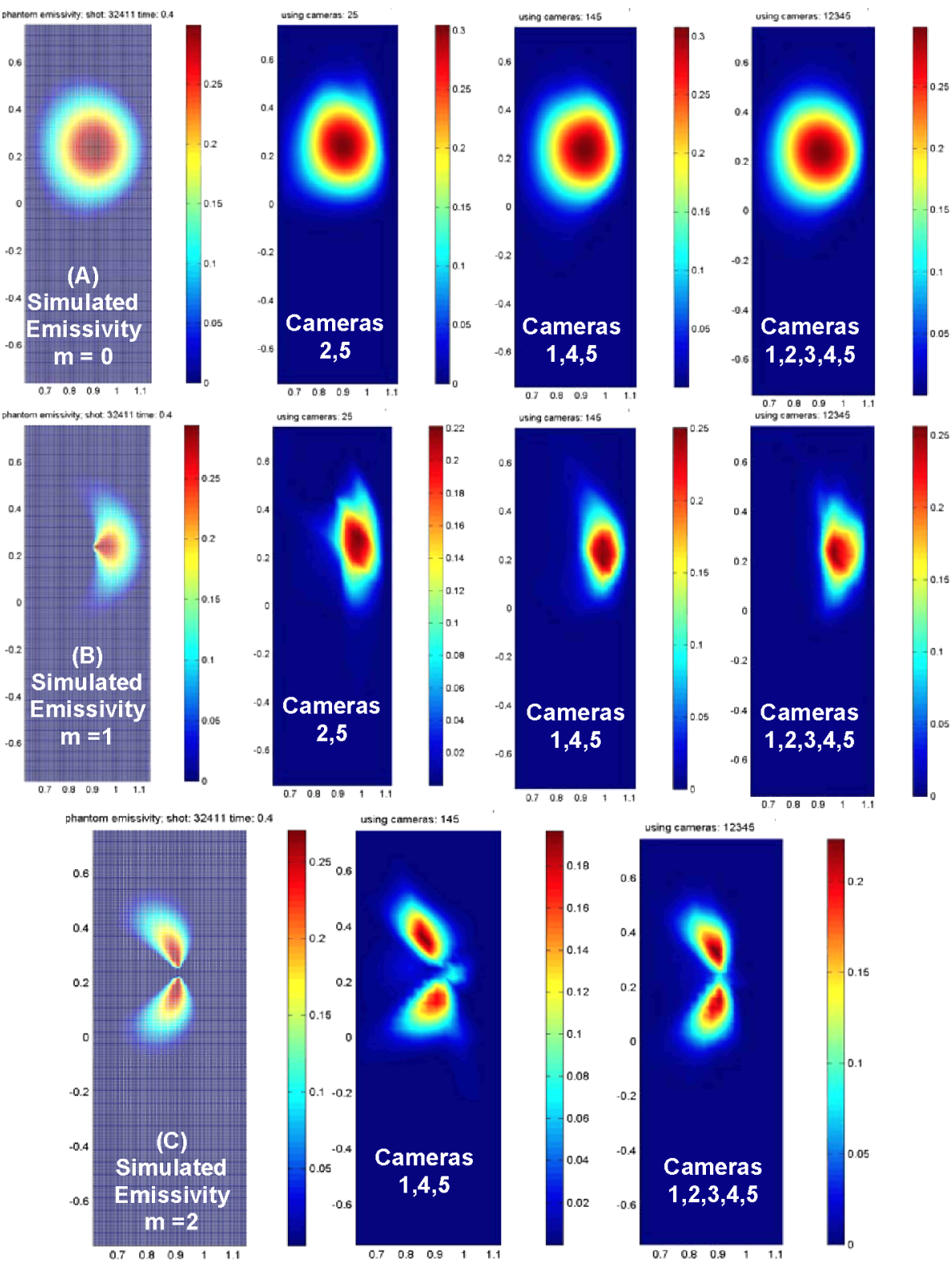

Figure 2. Tomographic reconstructions (2D contour plots in the poloidal plane) for three different postulated emissivity patterns: A) poloidally symmetric $(m=0)$ emissivity; $B) m=1$ emissivity; $C) m=2$ emissivity. The leftmost figure in each row is the phantom, followed by reconstructions with different camera combinations. The quality of the reconstruction increases by adding cameras viewing the plasma from different directions. 